University of Wollongong

Research Online

Faculty of Education - Papers (Archive)

Faculty of Arts, Social Sciences \& Humanities

$1-1-2009$

\title{
Using brain imaging to explore interactivity and cognition in multimedia learning environments
}

\author{
Barney Dalgarno \\ Charles Sturt University \\ Gregor Kennedy \\ University of Melbourne \\ Sue Bennett \\ University of Wollongong, sbennett@uow.edu.au
}

Follow this and additional works at: https://ro.uow.edu.au/edupapers

Part of the Education Commons

\section{Recommended Citation}

Dalgarno, Barney; Kennedy, Gregor; and Bennett, Sue: Using brain imaging to explore interactivity and cognition in multimedia learning environments 2009, 405-408.

https://ro.uow.edu.au/edupapers/116

Research Online is the open access institutional repository for the University of Wollongong. For further information contact the UOW Library: research-pubs@uow.edu.au 


\section{Using brain imaging to explore interactivity and cognition in multimedia learning environments}

\author{
Barney Dalgarno \\ Charles Sturt University \\ Boorooma Street, \\ Wagga Wagga, NSW 2678 \\ bdalgarno@csu.edu.au
}

\author{
Gregor Kennedy \\ University of Melbourne \\ Level 4, 766 Elizabeth Street, \\ Parkville, Melbourne, 3010 \\ gek@unimelb.edu.au
}

\author{
Sue Bennett \\ University of Wollongong \\ Wollongong NSW 2522 \\ sbennett@uow.edu.au
}

\begin{abstract}
Recent educational models of computer-based interactivity stress the important role of a learner's cognition. It has been suggested that interactive learning tasks carried out in the context of an authentic, problembased scenario will result in deeper elaborative cognitive processing leading to greater conceptual understanding of the material presented. Research methods that have been used to investigate cognition and learning have traditionally included self-report questionnaires, focus groups, interviews and think-aloud protocols and, more recently in computer-based settings, interaction log file or 'audit trail' analysis. While all of these techniques help researchers understand students' learning processes, all are limited in that they rely either on self-report or behavioural information to speculate about the cognitive activity of users. The use of functional brain imaging techniques has the potential to address this limitation. Drawing on issues encountered during a recent study using Functional Magnetic Resonance Imaging (fMRI), this paper discusses the methodological issues involved in the use of these techniques for exploring interactivity and cognition. Initial results comparing brain activation when exploring an interactive simulation with brain activation when using an equivalent tutorial program, for a single participant, are presented in order to provide information about the feasibility of the proposed methodological approach.
\end{abstract}

\section{Author Keywords}

interactivity, cognition, multimedia, functional brain imaging, fmri, learning

\section{ACM Classification Keywords}

H.1.2 User/Machine Systems

H.5. $\mathrm{m}$ Information interfaces and presentation:

Miscellaneous.

\section{INTRODUCTION}

This paper describes the research design and some initial data from a project exploring cognition in interactive multimedia learning environments using a combination of functional brain imaging and traditional behavioural and self-report measures. The paper begins with a discussion of the problem addressed and the traditional methods for

Permission to make digital or hard copies of part or all of this work or personal or classroom use is granted without fee provided that copies are not made or distributed for profit or commercial advantage and that copies bear this notice and the full citation on the first page. To copy otherwise, to republish, to post on servers, or to redistribute to lists, requires prior specific permission and/or a fee.

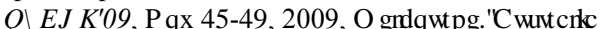

(C) ACM 2009 ISBN: 978-1-60558-प0ए-4/09/10 ...\$10.00 exploring it. It is then argued that functional imaging methods have promise in addressing aspects of the problem. The research questions and research design are then explained, including a discussion of the methodological issues encountered. Finally initial data from the first participant in the study are presented before discussing the methodological implications of the results obtained.

\section{INTERACTIVITY AND COGNITION RESEARCH}

For nearly 50 years researchers have been exploring the ways in which Computer-Assisted Learning (CAL) and more recently Interactive Multimedia (IMM) resources can contribute to learning. It is generally acknowledged that the key advantage such resources have over alternatives such as video, is the capacity for high levels of learner-computer interaction and engagement (Rieber, 2005). It has been suggested that interactive learning tasks carried out in the context of an authentic, problembased scenario will result in deeper, elaborative cognitive processing leading to greater conceptual understanding of the material presented (Rieber, 2005). A crucial focus of ongoing research has been the nature of the learnercomputer interaction and the connection between the different types of interaction and the desired learning processes and outcomes (Sims, 1997). More recently it has been acknowledged that any model of learnercomputer interaction must incorporate cognition as a central element. Put another way, it is the cognition that occurs through this interaction, rather than the behavioural activity, that is of central importance in predicting the learning that will occur (Dalgarno, 2004; Kennedy, 2004). Drawing on this body of prior research then, the aim of our research is to discover how interactivity in multimedia environments impacts on users' cognitive processes and subsequent learning outcomes.

Research methods that have been used to investigate cognition and learning have traditionally included observation, self-report questionnaires, focus groups, interviews and think-aloud protocols (Miles \& Huberman, 1994; Ericson \& Simon, 1993). In educational technology and human-computer interaction research these methods have been supplemented by the use of interaction log file or 'audit trail' analysis (Kennedy \& Judd, 2004). While all of these techniques help researchers understand students' learning processes, all are limited in that they rely either on self-report or behavioural information to speculate about the cognitive activity of users. In other words cognition is not directly measured. Consequently, although there is still a great 
deal that can be accomplished in addressing our research problem using these traditional methods, there may be value in also looking beyond these methods.

\section{THE ALTERNATIVE: FUNCTIONAL BRAIN IMAGING}

An alternative approach to exploring cognition is to use functional brain imaging methods, such as Functional Magnetic Resonance Imaging (fMRI) or Positron Emission Tomography (PET), to make inferences about the brain activation occurring during certain tasks. In recent years, with the increased availability of the equipment needed for these methods, the new field of cognitive neuroscience, which draws on physiological imaging techniques from neuroscience as well as behavioural techniques from psychology and theoretical approaches from cognitive science, has contributed to a range of problems previously explored only using behavioural methods (Churchland \& Sejnowski, 2000). Although functional brain imaging techniques have been used in neuroscience for more than 20 years, the widespread use of such techniques within psychology, cognitive science and education has only occurred within the last 10 years. There have, however, already been a significant number of published studies. Consequently, the equipment, materials and procedures are now well established.

Most of the research to date using functional brain imaging methods has focussed on the identification of brain regions activated while the participant undertakes a particular cognitive task (that is, with a goal of identifying the neural-correlates of these tasks). The tasks used in this research are typically very basic (verbal memory or simple problem solving tasks) because of the need to be precise about the nature of the brain activation associated with very specific tasks. This research has led to a large body of results associating brain areas with types of cognition.

This large body of data can potentially be drawn upon in interpreting the results of functional imaging studies involving more holistic tasks, such as problem-based learning tasks using interactive multimedia. For example, if a region of the brain associated with the storage of semantic information in long term memory is found to be activated to a greater extent during an interactive task than during attendance to the same information in a noninteractive fashion, then it could be concluded that the interactivity contributes to retention.

It is important to point out, however, that the cognitive neuroscience results to date have not established a one-toone relationship between cognitive tasks and brain areas. Cognitive tasks typically result in activation of a range of brain areas, and certain brain areas are activated by a range of different cognitive tasks. This is particularly the case for tasks involving higher order thinking. For example, any task involving problem solving will typically also involve storage and retrieval of information from working memory and often also from long-term memory. Nevertheless, we believe that there is sufficient data available to allow conclusions to be drawn about the degree to which brain activation data is consistent or inconsistent with accepted theories of learning. This can be done by comparing the cognition implied by brain activation measured during the use of interactive multimedia with the cognition proposed by theory.

\section{RESEARCH QUESTIONS}

This study, then, set out to explore the ways in which interactivity in multimedia environments impacts on users' cognitive processes and learning outcomes, using a combination of functional brain imaging and traditional methods. We were particularly interested in the types of learner-computer interaction occurring when a learner explores and manipulates a simulated environment, and consequently we decided to compare the learning processes involved in simulation-based and tutorial-based environments. The study attempted to address the following specific research questions:

- Is there a detectable difference in the overall brain activation between users of a simulation-based and a tutorial-based multimedia learning resource?

- If so, does this difference explain predicted differences in the learning processes and outcomes of users interacting with these two types of resources?

- Is brain activation during identified interactive episodes while using an educational multimedia resource consistent with the cognition predicted by theory?

Cognition and learning theory suggests that, compared to users of tutorial-based multimedia, users of simulationbased multimedia should experience greater degrees of elaborative processing and consequently form a greater number of semantic memory links through regularly drawing on their current understanding in making decisions and predicting how the simulated environment will respond (see Craik \& Lockhart, 1972; Wittrock, 1994; Norman \& Schmidt, 1992). Thus simulation users would be expected to show greater activation in areas of the brain associated with information manipulation and long-term memory storage and retrieval, compared to tutorial users. Such areas include the prefrontal cortex, especially the dorsolateral and ventrolateral areas, and the medial temporal lobe, especially the hippocampus (Fernandez \& Tendolker, 2001; Blumenfeld \& Ranganath, 2006; Prince, Daselaar \& Cabeza, 2005).

\section{RESEARCH DESIGN AND METHODOLOGICAL ISSUES}

The study used a combination of traditional data collection methods with functional brain imaging techniques. The participants' cognitive processing and learning outcomes in response to each type of stimulus material (simulation-based and tutorial-based) were explored using a range of data collection methods, including written pre- and post-tests on declarative knowledge and conceptual understanding; questionnaires on engagement and intrinsic motivation; audit trail methods to explore behavioural interactivity; stimulated response interviews involving the playback of the participant's recorded interactive session, in order to explore the participant's reflections on their own cognitive processing; and functional Magnetic Resonance 
Imaging (fMRI) to measure brain activation. After two pilot studies each involving a single participant, the main study was carried out using 8 participants.

It was originally intended that an existing multimedia resource would be used for the simulation condition. Each of the existing resources considered contained an interactive simulation as the central component, supplemented by text-based and graphical support materials. Such resources allow complete learner control over their exploration within the resource. The intention was to produce a tutorial resource based primarily on the text-based and graphical supplementary material within the resource, structured in a lock-step sequence with control only over the pace that the information was presented. However, a number of methodological issues emerged during our planning and pilot testing requiring us to depart substantially from this original intention. These issues included the following:

- Complex physical interaction intertwined with cognitive experimentation within the simulation condition could confound the results because it would be difficult to differentiate between brain activation associated with the motor tasks and brain activation associated with the cognitive task;

- Visual differences between the simulation and tutorial conditions could confound the results because it would be difficult to differentiate between the brain activation associated with attending to the rich multimedia content in the simulation condition from the activation associated with the cognitive task;

- It would be difficult to provide a regular baseline or rest stimulus within the simulation condition if we allowed complete learner control; and

- There was a need for each participant to use a simulation and a tutorial resource in turn so that brain activation comparisons could be done 'within' rather than 'between' participants (fMRI provides only relative activation information) and thus to avoid order effects there was a need for resources across two problem domains.

In addition to the methodological issues associated with using rich multimedia, we were also constrained by the fact that an MRI compatible mouse was not available to us. Because, it is unsafe to use any device that emits electromagnetic radiation in the scanner, special purpose devices using optical rather than electrical signals are required. Consequently, it was necessary to develop new resources or substantially tailor existing resources so that they used a push-button interface.

These issues were addressed primarily by designing new multimedia resources. Four resources were designed: a simulation and a tutorial based resource focussing on global warming, and a simulation and a tutorial based resource focussing on blood alcohol concentration. The following were the key features of the resources used in the study:

- Each resource was divided into two parts, a 'Background' section and a 'Main' section.
- The Background section, common to the simulation and tutorial versions, consisted of a series of screens containing background information about the problem domain which the participant moved backwards and forwards through.

- The Main section of the tutorial and simulation resources had identical screen layouts and both contained a regular baseline stimulus condition (a 'Rest Screen'), consisting of random numbers and graphs and an animated highlight.

- The Main section of the tutorial resources consisted of a series of 'Output Screens' showing the results of the simulation but without the ability to control the simulation parameters.

- The simulation resources were structured to isolate the different cognitive functions, with participants planning their manipulations on one screen (the 'Planning Screen'), carrying out their manipulations on another (the 'Manipulation Screen'), and viewing the output on a third (the 'Output Screen'), with the Rest Screen shown in between the Output and Planning screens during each cycle.

- Interaction occurred through the use of a device with three functional buttons. The left and right buttons moved a highlight forwards and backwards between options on the screen, and the middle button activated the highlighted option.

Figure 1 shows the 'Manipulation Screen' for the blood alcohol concentration simulation resource.

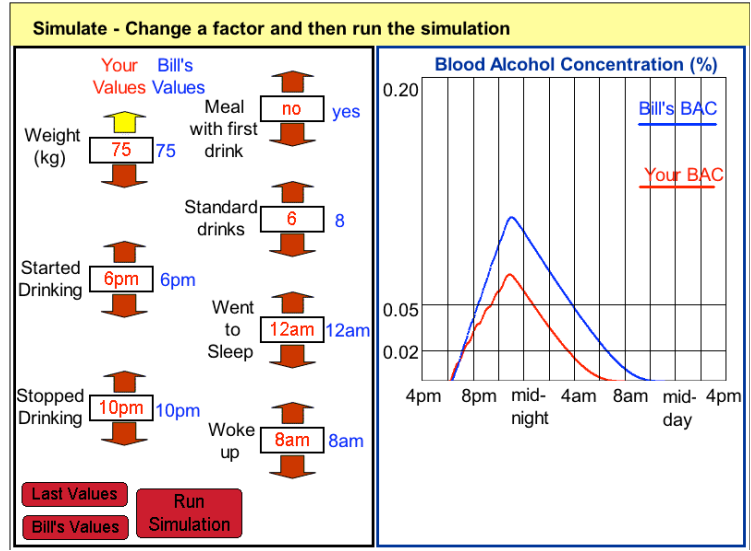

Figure 1. Blood Alcohol Simulation Manipulation Screen

\section{INITIAL RESULTS}

The results from one participant are discussed here. Of course the results of one participant will not allow us to respond to the research questions we have posed, but they will help to inform us about the appropriateness of the research design.

After carrying out a series of pre-processing steps including slice-timing correction, motion correction, and co-registration between the functional and structural images, voxel by voxel comparisons (T-tests) were carried out to compare the participant's activation across conditions. Table 1 summarises the results of some of these comparisons. The table shows the results of a series 
of T-tests comparing activation in specific brain areas across conditions or screens within the resources. The cognitive function normally associated with each brain area where activation differed is also briefly described (see, for example, Cabeza \& Nyberg, 2000).

\begin{tabular}{|l|l|l|}
\hline Comparison & $\begin{array}{c}\text { Brain areas } \\
\text { where activation } \\
\text { differed }\end{array}$ & \multicolumn{1}{|c|}{$\begin{array}{c}\text { Cognitive } \\
\text { function }\end{array}$} \\
\hline $\begin{array}{l}\text { Simulation: } \\
\text { Manipulation } \\
\text { Screen v Rest } \\
\text { Screen }\end{array}$ & $\begin{array}{l}\text { Areas of the } \\
\text { primary motor } \\
\text { cortex }\end{array}$ & $\begin{array}{l}\text { Control of the } \\
\text { right fingers and } \\
\text { both eyes }\end{array}$ \\
\hline $\begin{array}{l}\text { Simulation: } \\
\text { Output Screen } \\
\text { v Rest Screen }\end{array}$ & $\begin{array}{l}\text { Left and right } \\
\text { occipital lobe; } \\
\text { Left and right } \\
\text { dorsolateral } \\
\text { prefrontal cortex }\end{array}$ & $\begin{array}{l}\text { Visual } \\
\text { processing and } \\
\text { selection; } \\
\text { Manipulation of } \\
\text { information } \\
\text { during memory } \\
\text { encoding and/or } \\
\text { retrieval }\end{array}$ \\
\hline $\begin{array}{l}\text { Tutorial: } \\
\text { Output Screen } \\
\text { v Rest Screen }\end{array}$ & $\begin{array}{l}\text { Both sides of the } \\
\text { occipital lobe; } \\
\text { right dorsolateral } \\
\text { prefrontal cortex }\end{array}$ & As above \\
\hline $\begin{array}{l}\text { Simulation } \\
\text { Output Screen } \\
\text { v Tutorial } \\
\text { Output Screen }\end{array}$ & $\begin{array}{l}\text { Right parietal } \\
\text { lobe; Right } \\
\text { orbitofrontal and } \\
\text { frontopolar } \\
\text { prefrontal cortex }\end{array}$ & $\begin{array}{l}\text { Spatial working } \\
\text { memory; } \\
\text { Encoding of } \\
\text { abstract visual } \\
\text { information }\end{array}$ \\
\hline
\end{tabular}

Table 1. Summary of results for a single participant

\section{DISCUSSION AND CONCLUSION}

The results for the single participant summarised above are highly encouraging. The brain areas in which there are differential activations for each contrast are consistent with expectations. For example, the activations in the motor areas controlling the eyes and the right hand are exactly what would be expected on the Manipulation Screen indicating that the decision to isolate these activities on a single screen was effective. The activations on the simulation and tutorial Output Screens versus the Rest Screen are consistent with the idea that the learner would be carrying out cognitive manipulation of information, trying to relate the results to prior experience and then encoding and storing information for future retrieval. And the greater activation of a small area at the front of the prefrontal cortex when viewing the simulation Output Screen rather than the tutorial Output Screen is particularly encouraging, because it suggests, consistent with theory, deeper cognitive processing through the use of the simulation resource. Of course analysis of the remaining 7 participants' data will be necessary before clear conclusions can be drawn.

\section{ACKNOWLEDGMENTS}

Dr David Abbott and other staff from the Brain Research Institute for research design advice. Dr Michael Lew, University of Melbourne, who provided the blood alcohol concentration model. Charles Sturt University, who funded the project through a CSU Small Grant and the University of Wollongong Research Centre for
Interactive Learning Environments (RILE) who provided additional Seed Funding.

\section{REFERENCES}

Blumenfeld, R. S., \& Ranganath, C. (2006). Dorsolateral prefrontal cortex promotes long-term memory formation through its role in working memory organization. Journal of Neuroscience, 26(3), 916925.

Cabeza, R. \& Nyberg, L. (2000). Imaging cognition II: An empirical review of 275 PET and fMRI studies. Journal of Cognitive Neuroscience, 12(1), 1-47.

Churchland, P.S. and Sejnowski, T.J. (2000). In Michael S. Gazzaniga (Ed) Cognitive Neuroscience: A Reader. Malden, Mass: Blackwell.

Craik, F.I.M. \& Lockhart, R.S. (1972). Levels of processing: A framework for memory research. Journal of Verbal Learning and Verbal Behaviour, 11, 671-684.

Dalgarno, B. (2004). A classification scheme for learnercomputer interaction. In R.Atkinson, C.McBeath, D. Jones-Dwyer and R.Phillips (eds) Beyond the comfort zone, 21st annual conference ofASCILITE, (pp. 240-248). Perth, Australia.

Ericson, K. A., \& Simon, H. (1993). Protocol Analysis: Verbal Reports as Data. Cambridge, MA: MIT Press.

Fernández, G. \& Tendolkar, I. (2001). Integrated brain activity in medial temporal and prefrontal areas predicts subsequent memory performance: Human declarative memory formation at the system level. Brain Research Bulletin, 55(1), 1-9.

Kennedy, G. E. (2004). Promoting cognition in multimedia interactivity research. Journal of Interactive Learning Research, 15(1), 43-61.

Kennedy, G. E. \& Judd, T. S. (2004). Making sense of audit trail data. Australasian Journal of Educational Technology, 20(1), 18-32.

Miles, M. B., \& Huberman, A. M. (1994). Qualitative Data Analysis: An Expanded Sourcebook. Thousand Oaks, CA: Sage.

Norman, G. R. \& Schmidt, H. G. (1992). The psychological basis of problem-based learning: A review of the evidence. Academic Medicine, 67(9), 557-565.

Prince, S. E., Daselaar, S. M., \& Cabeza, R. (2005). Neural correlates of relational memory: Successful encoding and retrieval of semantic and perceptual associations. Journal of Neuroscience, 25(5), 1203 1210 .

Rieber, L.P. (2005). Multimedia learning in games, simulations and microworlds. In R.E.Mayer (Ed), The Cambridge Handbook of Multimedia Learning, NY: Cambridge University Press.

Sims, R. (1997). Interactivity: A forgotten art? Computers in Human Behavior, 13(2), 157-180.

Wittrock, M.C. (1994). Generative Science Teaching. In P.J. Fensham, R.F. Gunstone and R.T. White (eds), The Content of Science, (pp. 29-38). London: The Falmer Press. 